\title{
The Polarizing Power of the Interstellar Medium in Taurus
}

\section{Citation}

Arce, Héctor G., Alyssa A. Goodman, Pierre Bastien, Nadine Manset, and Matthew Sumner. 1998. "The Polarizing Power of the Interstellar Medium in Taurus." The Astrophysical Journal 499 (1): L93-97. https://doi.org/10.1086/311337.

\section{Permanent link}

http://nrs.harvard.edu/urn-3:HUL.InstRepos:41397435

\section{Terms of Use}

This article was downloaded from Harvard University's DASH repository, and is made available under the terms and conditions applicable to Other Posted Material, as set forth at http:// nrs.harvard.edu/urn-3:HUL.InstRepos:dash.current.terms-of-use\#LAA

\section{Share Your Story}

The Harvard community has made this article openly available.

Please share how this access benefits you. Submit a story.

Accessibility 


\title{
THE POLARIZING POWER OF THE INTERSTELLAR MEDIUM IN TAURUS
}

\author{
Héctor G. Arce, ${ }^{1,2}$ Alyssa A. Goodman, ${ }^{1,3}$ Pierre Bastien, ${ }^{4}$ Nadine Manset, ${ }^{4}$ and Matthew Sumner ${ }^{1,5}$ \\ Received 1997 December 5; accepted 1998 March 13; published 1998 May 6
}

\begin{abstract}
We present a study of the polarizing power of dust in cold dense regions (dark clouds) compared with that of dust in the general interstellar medium (ISM). Our study uses new polarimetric, optical, and spectral classification data for 36 stars to carefully study the relation between polarization percentage $(p)$ and extinction $\left(A_{V}\right)$ in the Taurus dark cloud complex. We find two trends in our $p-A_{V}$ study: (1) stars that are background to the warm ISM show an increase in $p$ with $A_{V}$, and (2) the percentage of polarization of stars that are background to cold dark clouds does not increase with extinction. We detect a break in the $p-A_{V}$ relation at an extinction of $1.3 \pm 0.2 \mathrm{mag}$, which we expect corresponds to a set of conditions where the polarizing power of the dust associated with the Taurus dark clouds drops precipitously. This break point places important restrictions on the use of polarimetry in studying interstellar magnetic fields.
\end{abstract}

Subject headings: dust, extinction — ISM: clouds — ISM: magnetic fields — polarization

\section{INTRODUCTION}

The polarization of background starlight has been used for nearly half a century to probe the magnetic field direction in the interstellar medium (ISM). The observed polarization is believed to be caused by dichroic extinction of background starlight passing through concentrations of aligned and elongated dust grains along the line of sight. Although there is no general consensus on which is the dominant grain alignment mechanism (Lazarian, Goodman, \& Myers 1997), it is generally believed that the shortest axis of the "typical" elongated grain tends to become aligned to the local magnetic field. For this orientation, the observed polarization vector is parallel to the plane-of-the-sky projection of a line-of-sight-averaged magnetic field (Davis \& Greenstein 1951).

The line-of-sight averaging inherent in background starlight polarimetric observations can make the interpretation of the polarization produced by different field orientations and/or several independent dust clouds very complicated. Nonetheless, it was thought that if lines of sight with just one localized, very dusty region (such as a dark cloud) between us and a background star could be found, surely the polarization would reveal the field associated with that dusty region. However, recent studies in the Taurus region (Goodman et al. 1992; Gerakines, Whittet, \& Lazarian 1995) and other parts of the sky (Creese, Jones, \& Kobulnicky 1995; Goodman et al. 1995) have uncovered substantial evidence to show that dust inside cold dark clouds has lower polarizing power than dust in the general warm ISM. This means that the polarization of the light from a background star is a nonuniformly weighted line-of-sight average of the projected plane-of-the-sky field and that grains in cold dark clouds are systematically down-weighted. The ultimate implication of this down-weighting is that above some (column?) density threshold, the polarization of background starlight gives no information about the magnetic field in dark

\footnotetext{
${ }^{1}$ Harvard-Smithsonian Center for Astrophysics, 60 Garden Street, Cambridge, MA 02138.

${ }^{2}$ National Science Foundation Minority Graduate Fellow.

${ }^{3}$ National Science Foundation Young Investigator.

${ }^{4}$ Université de Montréal, Département de Physique, and Observatoire du Mont Mégantic, C.P. 6128, Succ. Centre Ville, Montréal, Quebec H3C 3J7, Canada.

${ }^{5}$ Current address: California Institute of Technology, Department of Applied Physics, 116-81, Pasadena, CA 91125.
}

clouds. It is the goal of this Letter to find and physically describe this threshold.

The evidence that grains in cold dark clouds are inefficient polarizers of background starlight is multifaceted. Eight years ago, Goodman et al. (1990) found that the smooth large-scale patterns apparent in polarization maps of dark cloud complexes (e.g., Vrba, Strom, \& Strom 1976; Vrba, Coyne, \& Tapia 1981; Moneti et al. 1984; Whittet et al. 1994) do not systematically change in response to the large density enhancements represented by the dark clouds. After this realization, it was hypothesized that perhaps optical polarimetry was incapable of seeing field changes that might occur only in the high-density, optically opaque, interiors of dark clouds. So near-infrared polarimetry, which can probe the optically opaque cloud interiors, was undertaken. The near-infrared observations show that the mean direction and dispersion of the polarization vectors are virtually identical in the cloud interiors and their peripheries (Goodman et al. 1992, 1995). Thus, the presence of cold dark clouds still appeared to have no geometric effect on the polarization maps, implying either (1) that the field is truly unaffected by the cloud or (2) that background starlight polarimetry is somehow insensitive to the field in dark clouds. Polarization-extinction relations provide the best discriminant between these hypotheses. For grains of constant polarization efficiency, $p$ should rise with $A_{V}$. Using the near-infrared observations, Goodman et al. (1992, 1995) find that the percentage of polarization does not rise with extinction in cold dark clouds. The simplest interpretation ${ }^{6}$ of this result is that dust in dark clouds adds plenty to the observed extinction but has very little "polarizing power" and so adds only a very small fraction to the observed net polarization. A number of factors, including poor grain alignment, grain growth, and/or changes in grain shape or composition, could be responsible for the low polarization efficiency exhibited by dust grains in cold dark clouds (see Goodman et al. 1995). Regardless of which factor(s) cause(s) the low polarization efficiency exhib-

\footnotetext{
${ }^{6}$ Note that increased field tangling inside dark clouds cannot explain the near-infrared polarimetric observations for two reasons: (1) the dispersion in the distribution of position angle does not increase in the cloud interior (nearIR observations) relative to the periphery (optical observations), and (2) while it is true that the slope of a $p-A_{V}$ relation will diminish because of field tangling, it will remain positive even for highly tangled fields if all grains polarize equally well (see Jones 1989 and Jones, Klebe, \& Dickey 1992).
} 
ited by dust in dark clouds, the fact is that background starlight polarimetry does not reliably reveal the magnetic field in dark clouds.

Based on the near-infrared studies, we expect that the fraction of grains with high polarization efficiency is relatively constant in the lower density warm ISM but drops precipitously in dark clouds. Therefore, we hypothesize that a break point in the $p$ - $A_{V}$ relationship might exist at the dark clouds' "edges," which previous studies in the near-IR (Goodman et al. 1992, 1995; Gerakines et al. 1995) could not detect because of their inability to measure low $A_{V}$ 's accurately enough. In this Letter, we present our attempt to carefully study the $p-A_{V}$ relation near dark clouds, and thus we offer a set of guidelines as to where the polarization maps can be taken as faithful representations of the magnetic field projected onto the plane of the sky and where they cannot.

\section{DATA}

Our observing strategy consists of three parts. First, we obtain CCD images (using $B$ and $V$ broadband filters) of two $10^{\prime} \times \sim 5^{\circ}$ "cuts" (see Fig. 1 [Pl. L4]) through the Taurus dark cloud complex. Second, we measure the spectrum of 94 stars along the cuts in order to determine their spectral types. Using the multicolor photometry and the spectral types, we derive an extinction and distance to each of the stars, using the relation $A_{V}=R_{V} E_{B-V}$, with $R_{V}=3.1$ (Vrba \& Rydgren 1985). Third, we measure the polarization of as many of the stars for which we had extinction values based on the photometry and spectral classification, as time permits. (The polarimetry is by far the most time-consuming of the three steps.)

The cuts shown in Figure 1 pass at approximately right angles through two well-known, highly elongated dark clouds in the Taurus complex, L1506 and B216-217, both of which are thought to lie $140 \pm 10 \mathrm{pc}$ from the Sun (Kenyon, Dobrzycka, \& Hartman 1994). We chose this orientation for the cuts in order to ensure both (1) a sample of stars with a large dynamic range in extinction and (2) that many of the high- $A_{V}$ values measured would be produced by a single localized dark cloud along the line of sight. We attempted to exclude foreground stars by not selecting stars that appear relatively bright, and the measured spectroscopic parallax distances (see Arce \& Goodman 1998, hereafter AG98) confirm that we largely succeeded in doing so. (Our stellar sample has only three stars with distances less than $150 \mathrm{pc}$.) In total, the polarization of 31 stars was measured. In addition, we used previously published data to obtain color excesses for two stars (Kenyon et al. 1994) and polarization information for five stars (Goodman et al. 1990).

The broadband images were obtained using the CCD camera on the Smithsonian Astrophysical Observatory (SAO) Fred Lawrence Whipple Observatory (FLWO) $1.2 \mathrm{~m}$ telescope on Mount Hopkins, Arizona. The stellar spectra were obtained using the SAO FAST spectrograph on the FLWO $1.5 \mathrm{~m}$ telescope. The observations were carried out during the fall trimesters of 1995 and 1996. For information on the reduction of the photometric and spectroscopic data, see AG98. Employing an analysis method similar to that of Wood, Myers, \& Daugherty (1994), we also created an extinction map of Taurus, with $5^{\prime}$ resolution, using co-added images of flux density from the IRAS Sky Survey Atlas (see AG98).

The polarization observations were carried out at the Mont Mégantic Observatory, using the Beauty and The Beast twochannel photoelectric polarimeter (described in Manset \& Bastien 1995) on the $1.6 \mathrm{~m}$ Ritchey-Chrétien telescope, in 1996
February, December, and 1997 February. We used a Schott RG-645 filter that, combined with the photomultiplier response, gives a broad passband of $2410 \AA$, centered at $7660 \AA$. A small instrumental polarization of $\sim 0.1 \%$ was measured and subtracted from the observations. The polarization scale was determined by observing a bright star and using a calibration prism that gives essentially $100 \%$ polarized light. The origin of the position angles was determined by observing standard polarized stars. The uncertainty in the polarization angle $(\theta)$ is given by the formula $\sigma(\theta)=28.65[\sigma(p) / p]$ (Serkowski 1974).

Table 1 summarizes the relevant polarization and extinction values derived from all of the data collected and assembled.

\section{RESULTS}

Plots of polarization versus extinction are shown in Figure 2. There seem to be two different trends in the $p-A_{V}$ plots. Most stars with $A_{V} \lesssim 1.3$ mag follow a trend in which their percentage of polarization increases with extinction. But many stars with $A_{V} \gtrsim 1.3$ mag keep a more or less constant $p$ with extinction. By overlaying the stars' positions on an extinction map (Fig. 1), it becomes apparent that several of the stars that have high extinction but low polarization are very near to peaks in the optical depth (i.e., dark clouds). On the other hand, the positions of most of the other stars that follow an increasing $p$ with increasing $A_{V}$ tend to lie either on places where the optical depth is small (like the southernmost stars) or on small, randomly occurring, high-extinction patches, far away from the optical depth peaks.

From the results of previous studies, we can easily guess that the $p-A_{V}$ relation for stars that are background to cold dark clouds might be different from that of stars background to the general (lower density) ISM. Thus, it makes sense to attempt to separate our stellar sample systematically into stars that are and are not "background to dark clouds," in order to study the potentially different $p-A_{V}$ relations. Visually inspecting the positions of the stars superposed on an extinction map (see Fig. 1) is not an adequately quantitative method of reliably achieving this separation. Our definition of a star that is "background to a dark cloud" is one that lies close to an extinction peak and is primarily extinguished by dust in dense regions at a single distance. With this definition in mind, we use two different means of differentiating between the two "types" of stars.

The first method uses the IRAS $A_{V}$ map. The three main extinction ridges that cross the slice of Taurus shown in Figure 1 are L1506, B216-217, and the region that Wood et al. (1994) name IRAS core Tau M1. For each star that lies near one of these ridges, we plot $A_{V}$ along a line on the plane of the sky defined by the normal to the nearest ridge from the star. For each of these $A_{V}$ versus offset profiles, we fitted a Gaussian to the resulting extinction profile and then measured the distance from the $A_{V}$ peak to the star's position, in units of half-width at half-maximum (HWHM). The results of this procedure are shown in Table 1. We then divide the sample into two groups: stars that appear close to the optical depth peaks in projection and stars that appear far away. We experimented with different distances as the boundary between the two groups and decided finally to adopt 1.6 HWHM as such. With 1.6 HWHM as the dividing value, we obtain two clear $p-A_{V}$ trends in the sample, while having the dividing distance between close and far stars from the dark cloud extinction peaks as far as possible.

The second method of separating the stars uses the fact that the observed extinction can be thought of as a sum of the extinction due to the dense regions of the dark cloud and the extinction due to the dust foreground and background to the 
TABLE 1

Polarization Data

\begin{tabular}{|c|c|c|c|c|c|c|}
\hline Star Name ${ }^{a}$ & $\begin{array}{c}p \\
(\%)\end{array}$ & $\begin{array}{c}\theta \\
\text { (east of north) }\end{array}$ & $\begin{array}{c}A_{V}^{\mathrm{b}} \\
(\mathrm{mag})\end{array}$ & HWHM $^{c}$ & $\begin{array}{c}\text { Fraction of IRAS } A_{V}^{\mathrm{d}} \\
(\%)\end{array}$ & Comment $^{\mathrm{e}}$ \\
\hline 011005 & $1.11 \pm 0.24$ & $79.2 \pm 6.2$ & $0.73 \pm 0.16$ & $\ldots$ & $\ldots$ & No \\
\hline 021013 & $0.71 \pm 0.34$ & $56.5 \pm 13.7$ & $0.72 \pm 0.23$ & $\ldots$ & $\ldots$ & No \\
\hline $021012 \ldots \ldots \ldots$ & $0.35 \pm 0.31$ & $41.0 \pm 25.7$ & $0.64 \pm 0.16$ & $\ldots$ & $\ldots$ & No \\
\hline $021011 \ldots \ldots \ldots$ & $0.89 \pm 0.39$ & $35.9 \pm 12.5$ & $0.73 \pm 0.16$ & $\ldots$ & $\ldots$ & No \\
\hline $031023 \ldots \ldots \ldots$ & $0.13 \pm 0.23$ & $98.3 \pm 51.1$ & $0.70 \pm 0.16$ & $\ldots$ & $\ldots$ & No \\
\hline $041033 \ldots \ldots \ldots$ & $2.16 \pm 0.45$ & $41.6 \pm 5.9$ & $1.59 \pm 0.27$ & 2.3 & 14 & No \\
\hline 041032 & $1.51 \pm 0.24$ & $61.4 \pm 4.6$ & $1.50 \pm 0.23$ & 1.4 & 30 & Yes \\
\hline $051041 \ldots \ldots \ldots$ & $1.09 \pm 0.14$ & $63.2 \pm 3.7$ & $1.08 \pm 0.17$ & $\ldots$ & $\ldots$ & No \\
\hline 071064 & $1.33 \pm 0.08$ & $70.3 \pm 1.8$ & $1.37 \pm 0.15$ & $\ldots$ & $\ldots$ & No \\
\hline $071062^{\mathrm{f}}$ & $2.08 \pm 0.08$ & $70.4 \pm 1.1$ & $1.74 \pm 0.30$ & $\ldots$ & $\ldots$ & No \\
\hline 081075 & $1.48 \pm 0.25$ & $75.5 \pm 4.9$ & $1.65 \pm 0.26$ & 3.0 & 0.7 & No \\
\hline AG $136^{\mathrm{g}}$ & $1.60 \pm 0.10$ & $83.0 \pm 2.0$ & $1.33 \pm 0.15$ & 1.5 & 27 & Yes \\
\hline AG $133^{\mathrm{g}} \ldots \ldots \ldots$ & $1.83 \pm 0.11$ & $76.0 \pm 2.0$ & $1.27 \pm 0.18$ & 2.2 & 9 & No \\
\hline AG $132^{\mathrm{g}} \ldots \ldots \ldots$ & $1.37 \pm 0.18$ & $7.0 \pm 4.0$ & $1.67 \pm 0.19$ & 1.6 & 20 & Yes \\
\hline AG $102^{\mathrm{g}}$ & $2.10 \pm 0.06$ & $99.0 \pm 1.0$ & $3.10 \pm 0.34$ & 0.9 & 34 & Yes \\
\hline 091084 & $0.99 \pm 0.65$ & $101.2 \pm 18.5$ & $2.27 \pm 0.24$ & 0.9 & 40 & Yes \\
\hline 092087 & $1.14 \pm 0.13$ & $96.4 \pm 3.2$ & $2.45 \pm 0.17$ & 1.6 & 21 & Yes \\
\hline AG $105^{\mathrm{g}}$ & $0.93 \pm 0.11$ & $85.0 \pm 3.0$ & $1.58 \pm 0.24$ & 2.1 & 4 & No \\
\hline 091081 & $2.18 \pm 0.40$ & $28.8 \pm 5.3$ & $1.11 \pm 0.15$ & $\ldots$ & $\ldots$ & No \\
\hline 101095 & $2.07 \pm 0.45$ & $45.6 \pm 6.3$ & $1.34 \pm 0.16$ & $\ldots$ & $\ldots$ & No \\
\hline 101094 & $1.21 \pm 0.18$ & $55.5 \pm 4.3$ & $1.35 \pm 0.15$ & $\ldots$ & $\ldots$ & No \\
\hline $\mathrm{S} 76573^{\mathrm{fth}}$ & $0.03 \pm 0.08$ & $139.5 \pm 57.7$ & $0.00 \pm 0.15$ & $\ldots$ & $\ldots$ & No \\
\hline $101091 \ldots \ldots \ldots$ & $2.16 \pm 0.22$ & $60.6 \pm 2.9$ & $1.57 \pm 0.16$ & $\ldots$ & $\ldots$ & No \\
\hline $111104 \ldots \ldots \ldots$ & $0.46 \pm 0.12$ & $96.5 \pm 7.6$ & $1.41 \pm 0.15$ & $\ldots$ & $\ldots$ & No \\
\hline $111101 \ldots \ldots \ldots$ & $1.51 \pm 0.08$ & $43.6 \pm 1.5$ & $1.80 \pm 0.35$ & $\ldots$ & $\ldots$ & No \\
\hline $121115 \ldots \ldots \ldots$ & $1.39 \pm 0.18$ & $55.4 \pm 3.7$ & $1.78 \pm 0.22$ & $\ldots$ & $\ldots$ & No \\
\hline $121113 \ldots \ldots \ldots$ & $2.75 \pm 0.32$ & $49.4 \pm 3.3$ & $1.45 \pm 0.22$ & $\ldots$ & $\ldots$ & No \\
\hline $131123 \ldots \ldots \ldots$ & $2.41 \pm 0.29$ & $60.0 \pm 3.4$ & $1.85 \pm 0.26$ & 1.2 & 42 & Yes \\
\hline 131121 & $1.44 \pm 0.28$ & $21.1 \pm 5.7$ & $1.83 \pm 0.23$ & 0.3 & 41 & Yes \\
\hline $141135 \ldots \ldots \ldots$ & $1.54 \pm 0.21$ & $37.9 \pm 3.9$ & $2.29 \pm 0.16$ & 0.2 & 40 & Yes \\
\hline $141134 \ldots \ldots \ldots$ & $1.47 \pm 0.09$ & $43.3 \pm 1.8$ & $3.73 \pm 0.16$ & 0.2 & 40 & Yes \\
\hline $141133 \ldots \ldots \ldots$ & $2.70 \pm 0.25$ & $28.2 \pm 2.7$ & $1.76 \pm 0.15$ & 0.9 & 23 & Yes \\
\hline 00B2.2 $\ldots \ldots \ldots$ & $1.57 \pm 0.14$ & $11.1 \pm 2.5$ & $2.03 \pm 0.16$ & 1.5 & 33 & Yes \\
\hline 00B2.1 & $2.08 \pm 0.13$ & $17.1 \pm 1.9$ & $1.35 \pm 0.16$ & 2.6 & 1 & No \\
\hline TDC $321 \ldots \ldots$ & $2.87 \pm 0.17$ & $12.3 \pm 1.7$ & $2.87 \pm 0.17$ & $\ldots$ & $\ldots$ & No \\
\hline S76574 f,h $\ldots \ldots$ & $2.60 \pm 0.08$ & $10.2 \pm 1.0$ & $1.46 \pm 0.15$ & $\ldots$ & $\ldots$ & No \\
\hline
\end{tabular}

${ }^{a}$ The stars are ordered in increasing declination, and the coordinates are given in AG98.

${ }^{\mathrm{b}}$ The error in $A_{V}$ does not include the error introduced by assuming a constant value of $R_{V}$.

${ }^{\mathrm{c}}$ Distance from nearest dark cloud $A_{V}$ peak to the star's position in units of HWHM of the fitted Gaussian.

${ }^{d}$ Percentage of the star's total IRAS $A_{V}$ that is due to the baseline-subtracted Gaussian.

${ }^{\mathrm{e}}$ Was the star classified as being background to a dark cloud?

${ }^{\mathrm{f}}$ Stars with a distance less than $150 \mathrm{pc}$.

g Polarization data obtained from Goodman et al. 1990.

${ }^{\mathrm{h}}$ These are SAO stars. Their photometry, spectral classification, and distance were obtained from Kenyon et al. 1994.

dark cloud. Using the same extinction profiles determined from the IRAS map in the first method, we assume that by subtracting a baseline from the Gaussian, we can eliminate the extinction due to the dust foreground and background to the cloud, and that the baseline-subtracted Gaussian gives an estimate of the extinction due to the dense region of the cloud. The value of the baseline-subtracted Gaussian at the stars' projected position can then be compared with the total value of IRAS $A_{V}$ at the same position, giving an estimate of the percentage of extinction due to the dark cloud itself. We say that a star is "background to a dark cloud" if more than $20 \%$ of the IRAS $A_{V}$ at the star's projected position is due to the baseline-subtracted Gaussian. A potential problem with this method is that the dark cloud does not necessarily have to have a Gaussian profile-it could be Gaussian-like, with broad wings. If this is so, then by subtracting a baseline, one is potentially subtracting a substantial part of the extinction due to the dark cloud.

We classify a star as being "background to a dark cloud" if it satisfies the background star criteria for both of the two different methods (see Table 1).

Figure 2 clearly shows that $p$ increases with $A_{V}$ for stars that were classified as being background to the ISM, while $p$ is roughly constant with $A_{V}$ for stars classified as being background to dark clouds. After making linear least-square fits, weighted by the uncertainty in $p$, to the two different groups, we find that the trends cross at $A_{V}=1.3 \mathrm{mag}$. The errors in the fitted line coefficients imply an uncertainty of $\pm 0.2 \mathrm{mag}$ for this intersection point. Thus, we conclude that there is a break in the $p-A_{V}$ relation for this region of Taurus at $A_{V}=$ $1.3 \pm 0.2$ mag.

We believe that choosing a constant value of $R_{V}$ to derive the extinction to each star does not adversely affect our results. Studies have shown that denser regions usually harbor bigger grains (Whittet \& Blades 1980) and that bigger grains imply larger values of $R_{V}$ (Cardelli, Clayton, \& Mathis 1989), which in turn imply a greater value of $A_{V}$ for a given value of $E_{B-V}$. Thus, we expect that if there are changes in $R_{V}$ toward different lines of sight, then $R_{V}$ should be larger for lines of sights that pass through dark clouds. If this is so, then the stars observed through these lines of sight would have more extinction than originally calculated. These points would then shift to the right in our $p$ versus $A_{V}$ plot in Figure 2. If this happened, the break in the $p-A_{V}$ relation would just be more pronounced and clear. We are relatively certain that the value of $R_{V}$ does not vary in 


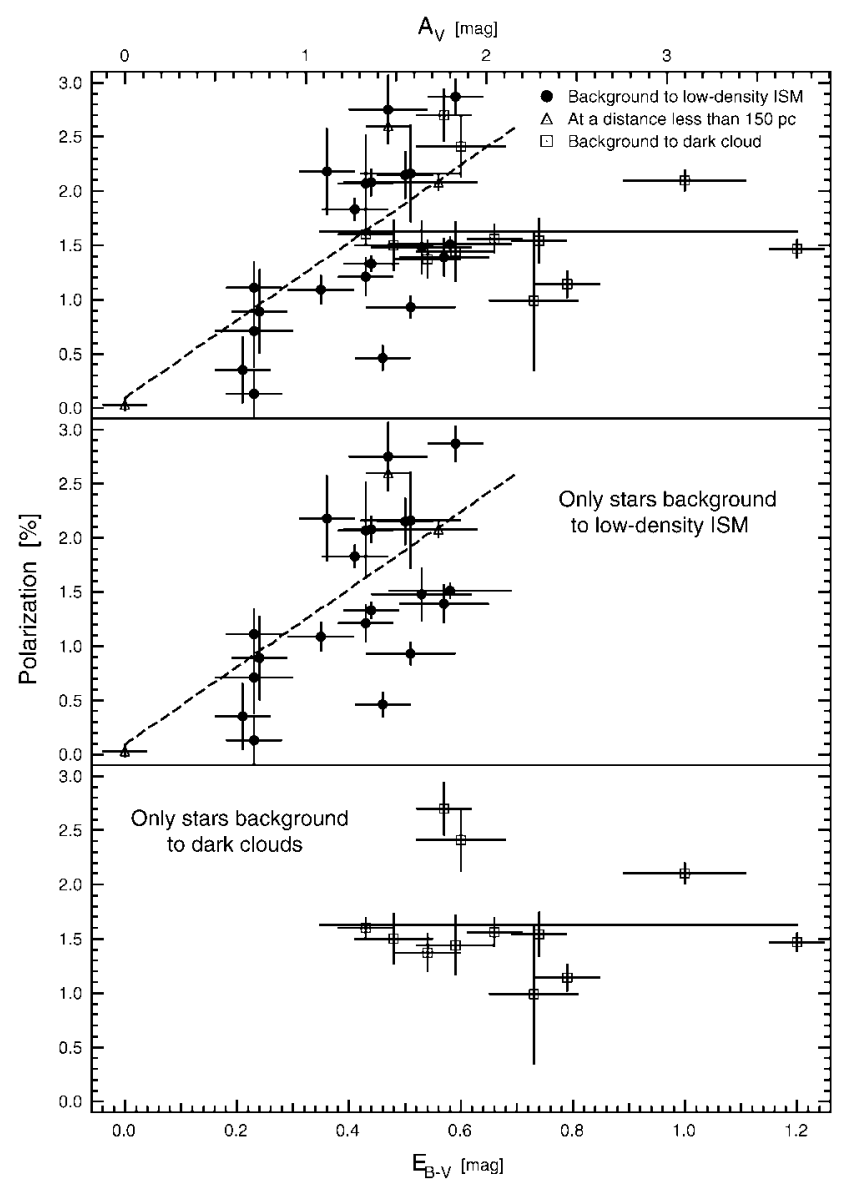

FIG. 2.-Observed relation between polarization and extinction, where $A_{V}=3.1 E_{B-V}$. The lines are least-square linear fits, weighted by the uncertainty in $p$. The dashed line is the fit to the points representing the stars background to the low-density ISM, which gives $p=(0.09 \pm 0.06)+(3.58 \pm 0.13) E_{B-V}$, with a correlation coefficient of 0.68 . The solid line is the fit to the points representing the stars background to dark clouds, which gives $p=(1.61 \pm$ $0.13)+(0.03 \pm 0.15) E_{B-V}$, with a correlation coefficient of 0.79 .

a systematic way for stars that are background to the general ISM, since their color-excess-determined extinction agrees very well with the other methods of obtaining extinction (see AG98).

\section{ANALYSIS AND DISCUSSION}

The two trends that we find in our data-rising $p$ with $A_{V}$ and roughly constant $p$ with $A_{V}$-have each been observed in previous studies. But these trends have never been observed together, in the same region, as clearly distinct trends before. In fact, most studies find very large scatter in their $p-A_{V}$ plots, and a clear linear correlation in the $p-A_{V}$ relation for stars that are background to warm dust-our first trend-is observed in only a few studies, such as Wilking et al. (1979) or Guetter \& Vrba (1989). Theoretically, one should observe this linear correlation if, along all lines of sight, each grain polarizes light equally well and there are no big changes in the field orientation. Most probably this is the case in these two studies since most of their lines of sight pass through similar environments, in the small region of the sky that they studied. The second trend in our data - a slow rise or no rise in $p$ with $A_{V}$ - has been observed in near-IR polarimetric studies of stars that are background to cold dark clouds (Goodman et al. 1992, 1995).

In general, most studies of the $p-A_{V}$ relation find that there is a fuzzy upper bound to the amount of polarization possible for a particular extinction and that observed points lie anywhere below this upper limit in the $p-A_{V}$ plane (Hiltner 1956; Vrba et al. 1976, 1981, 1993; Moneti et al. 1984; Whittet et al. 1994; Gerakines et al. 1995). Such results can easily be explained by allowing the polarization efficiency of the grains and/or the field orientation with respect to the plane of the sky to vary from one line of sight to another (Hiltner 1956). Polarization studies of regions near dark clouds most probably sample lines of sight passing through a variety of different physical environments-lines of sight with different dust and gas densities, which likely contain grains with different average polarizing efficiencies. In most cases, the samples in these studies are not separated depending on the characteristics of the line of sight; thus, the upper-envelope behavior exhibited by their $p-A_{V}$ relations is expected. Moreover, many of these studies also find a decrease in polarization efficiency $(p / A)$ with increasing extinction, which is likely due to the fact that the highly extinguished stars often lie behind dark clouds, where dust is likely to have a lower polarization efficiency.

A break in the $p-A_{V}$ relation can be predicted for stars that are background to quiescent dark clouds by many different theories. Most recently, Draine \& Weingartner (1996) studied the effect of radiative torques due to the anisotropic illumination of helical grains. They came to the conclusion that, in the average ISM, diffuse cloud, and warm star-forming region, such a process is able to produce rotational velocities higher than the ones produced by suprathermal mechanisms studied previously (e.g., spin-up caused by $\mathrm{H}_{2}$ formation on grains; Purcell 1979). But, on the other hand, they conclude that inside quiescent dark clouds, the radiative torques are unimportant because of the weakened radiation fields and higher matter density. They explicitly state that they expect grain alignment near the cloud surface but not at depths of $A_{V} \gtrsim 2$ mag. Moreover, a quantitative study of six types of alignment mechanisms in the dark cloud L1755 was done by Lazarian et al. (1997). Using data from the literature, they studied the joint action of different alignment mechanisms in the outer, intermediate, and inner regions of the cloud. They came to the conclusion that all the major mechanisms fail to produce alignment in the inner and intermediate regions (which they assume to have $A_{V} \gg 1$ ), while grain alignment is efficient in the outer regions $\left(A_{V} \lesssim 1\right)$. The value we obtain for the break in the $p-A_{V}$ relation, $A_{V}=1.3 \pm 0.2 \mathrm{mag}$, is consistent with these two studies. These results are not necessarily inconsistent with the observed polarization in the $3 \mu \mathrm{m}$ ice feature (Hough et al. 1988). Polarization in the ice feature is only observed in stars that are background to star-forming clouds ( $\mathrm{HCl} 2, \mathrm{OMC}-1)$, which are more likely warmer than the filamentary cold dark clouds in our study and hence can harbor grains with higher polarization efficiency (see Fig. 7 in Goodman et al. 1995).

\section{CONCLUSION}

The break point in the $p-A_{V}$ relation places important restrictions on the use of polarimetry in studying interstellar magnetic fields. Since the polarization efficiency of the dust inside dark clouds is very low, most of the polarization observed for lines of sight that pass through these extinction peaks is not due to the dark cloud but is due to dust that is background and foreground to the cloud. Hence, one should not use background starlight polarimetry to map magnetic fields inside dark clouds. With the results of this study, we can quantify the word "inside." In regions like Taurus, it is safe to interpret the polar- 
ization of background starlight as a representation of the planeof-the-sky-projected magnetic field up to the $1.3 \pm 0.2 \mathrm{mag}$ "edge" of the dark cloud. In other words, the linear relation between $p$ and $A_{V}$ that exists in the low-density ISM breaks down for stars that are background to the $\gtrsim 1.3$ mag of extinction produced by a dense localized dusty region (i.e., dark cloud). After this edge, polarization no longer rises with extinction, and thus it cannot reveal the field structure in the dense region. We restate that this proscription applies only for stars that are background to cold dark clouds, since stars that are background to the warm ISM have not been shown to exhibit such behavior.

H. G. A. gratefully acknowledges the support from the National Science Foundation Minority Graduate Fellowship. We would like to extend our gratitude to Scott J. Kenyon, Lucas M. Macri, and Perry Berlind for their great help in the photometry analysis, spectral classification of stars, and acquisition of data.

\section{REFERENCES}

Arce, H. G., \& Goodman, A. A. 1998, in preparation (AG98)

Cardelli, J. A., Clayton, G. C., \& Mathis, J. S. 1989, ApJ, 345, 245

Creese, M., Jones, T. J., \& Kobulnicky, H. A. 1995, AJ, 110, 268

Davis, L., \& Greenstein, J. L. 1951, ApJ, 114, 206

Draine, B. T., \& Weingartner, J. C. 1996, ApJ, 470, 551

Gerakines, P. A., Whittet, D. C. B., \& Lazarian, A. 1995, Astrophys. Lett., 455,171

Goodman, A. A., Bastien, P., Myers, P. C., \& Ménard, F. 1990, ApJ, 359, 263

Goodman, A. A., Jones, T. J., Lada, E. A., \& Myers, P. C. 1992, ApJ, 399, 108

. 1995, ApJ, 448, 748

Guetter, H. H., \& Vrba, F. J. 1989, AJ, 98, 611

Hiltner, W. A. 1956, ApJS, 2, 389

Hough, J. H., et al. 1988, MNRAS, 230, 107

Jones, T. J. 1989, ApJ, 346, 728

Jones, T. J., Klebe, D., \& Dickey, J. M. 1992, ApJ, 422, 164

Kenyon, S. J., Dobrzycka, D., \& Hartman, L. 1994, AJ, 108, 1872
Lazarian, A., Goodman, A. A., \& Myers, P. C. 1997, ApJ, 490, 273

Manset, N., \& Bastien, P. 1995, PASP, 107, 483

Moneti, A., Pipher, J. L., Helfer, H. L., McMillan, R. S., \& Perry, M. L. 1984, ApJ, 282, 508

Purcell, E. 1979, ApJ, 231, 404

Serkowski, K. 1974, in Methods of Experimental Physics, Vol. 12: Astrophysics, Part A, ed. N. Carleton (New York: Academic), 361

Vrba, F. J., Coyne, G. V., \& Tapia, S. 1981, ApJ, 243, 489 . 1993, AJ, 105, 1010

Vrba, F. J., \& Rydberg, A. E. 1985, AJ, 90, 1490

Vrba, F. J., Strom, S. E., \& Strom, K. M. 1976, AJ, 87, 956

Whittet, D. C. B., \& Blades, J. C. 1980, MNRAS, 190, 41

Whittet, D. C. B., Gerakines, P. A., Carkner, A. L., Hough, J. H., Martin, P. G., Prusti, T., \& Kilkenny, D. 1994, MNRAS, 268, 1

Wilking, B. A., Lebofsky, M. J., Kemp, J. C., \& Rieke, G. H. 1979, AJ, 84, 199

Wood, D., Myers, P. C., \& Daugherty, D. A. 1994, ApJS, 95, 457 


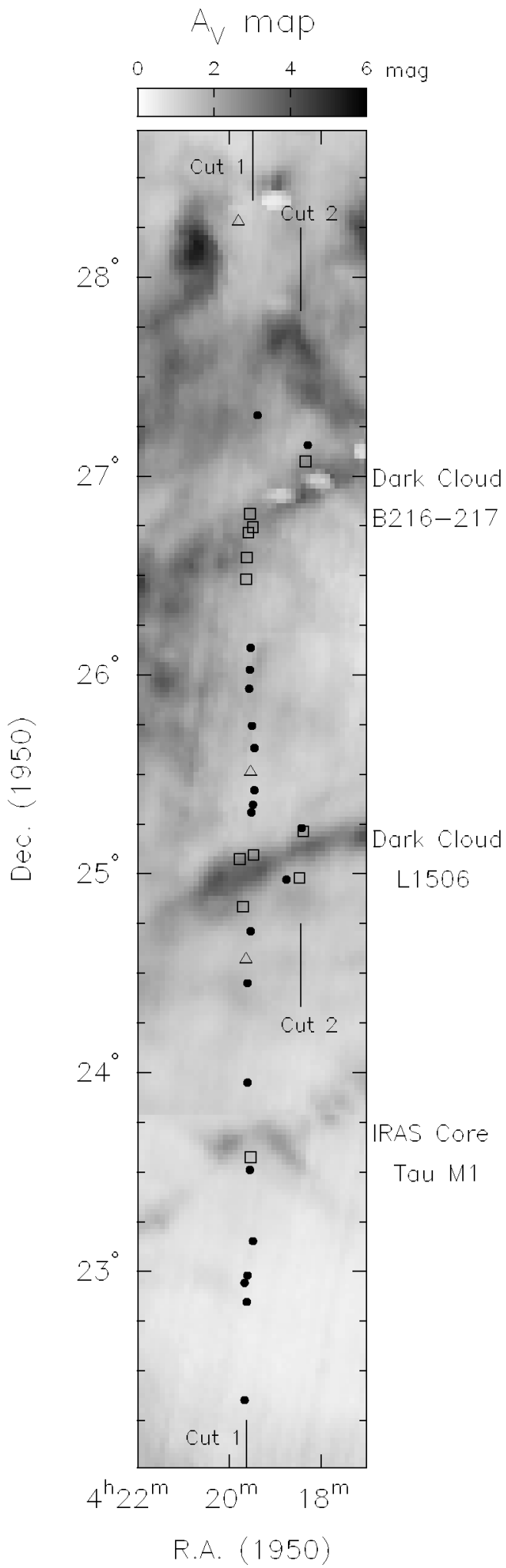

FIG. 1.-Extinction $\left(A_{V}\right)$ map of the region under study, obtained by employing an analysis method similar to that of Wood et al. (1994), using co-added images of flux density from the IRAS Sky Survey Atlas (see AG98). The positions of the 36 stars in our sample are shown: stars background to the low-density ISM (filled circles); stars with a distance of less than $150 \mathrm{pc}$ (open triangles); stars classified as "background to dark clouds" (open squares). The lines mark the positions of the two cuts.

ArCE et al. (see 499, L94)

PLATE L4 